\title{
Outage probability analysis for hybrid TSR-PSR based SWIPT systems over log-normal fading channels
}

\author{
Hoang Thien Van ${ }^{1}$, Hoang-Phuong Van ${ }^{2}$, Danh Hong Le ${ }^{3}$, Ma Quoc Phu ${ }^{4}$, Hoang-Sy Nguyen ${ }^{5}$ \\ ${ }^{1}$ The Saigon International University (SIU), Ho Chi Minh City, Vietnam \\ ${ }^{2}$ Faculty of Engineering and Technology, Thu Dau Mot University, Thu Dau Mot City, Binh Duong Province, Vietnam \\ ${ }^{3}$ Van Hien University, Ho Chi Minh City, Vietnam \\ ${ }^{4,5}$ Binh Duong University, Thu Dau Mot City, Binh Duong Province, Vietnam \\ ${ }^{5}$ Eastern International University, Binh Duong Province, Vietnam
}

\section{Article Info}

Article history:

Received Jul 19, 2019

Revised Mar 26, 2021

Accepted Apr 6, 2021

\section{Keywords:}

Energy harvesting

Log-normal fading

Outage probability

Power splitting relaying

Time switching relaying

\begin{abstract}
Employing simultaneous information and power transfer (SWIPT) technology in cooperative relaying networks has drawn considerable attention from the research community. We can find several studies that focus on Rayleigh and Nakagami-m fading channels, which are used to model outdoor scenarios. Differing itself from several existing studies, this study is conducted in the context of indoor scenario modelled by log-normal fading channels. Specifically, we investigate a so-called hybrid time switching relaying (TSR)-power splitting relaying (PSR) protocol in an energy-constrained cooperative amplify-and-forward (AF) relaying network. We evaluate the system performance with outage probability (OP) by analytically expressing and simulating it with Monte Carlo method. The impact of power-splitting (PS), time-switching (TS) and signal-to-noise ratio (SNR) on the OP was as well investigated. Subsequently, the system performance of TSR, PSR and hybrid TSR-PSR schemes were compared. The simulation results are relatively accurate because they align well with the theory.
\end{abstract}

This is an open access article under the CC BY-SA license.

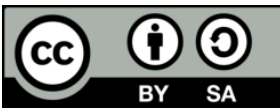

Corresponding Author:

Hoang-Van Phuong

Faculty of Engineering and Technology

Thu Dau Mot University

Thu Dau Mot City, Binh Duong Province, Vietnam

Email: phuongvh@tdmu.edu.vn

\section{INTRODUCTION}

For conventional energy-constrained wireless networks, it is occasionally hard or even impossible to recharge or replace batteries [1], [2]. Instead, we can exploit the natural resources (wind energy, solar energy) by EH modules to keep the networks energized, although with some limitations because of their fluctuating properties [3], [4]. Thereby, the research community has developed the simultaneous information and power transfer (SWIPT) technology to alter the renewable energy with radio frequency (RF), [5]-[8].

Rayleigh fading channels have been utilized for studies about the adaptive relaying protocol of twohop amplify-and-forward (AF) relaying networks with exceptional throughput performance [9], [10]. Besides, in [11] where the outage probability (OP) of decode-and-forward (DF) PSR system was analyzed, we can see that EH relaying network is more beneficial than conventional networks. Additionally, it is essential to deploy hybrid concepts to study and effectively improve system performance. For example, from the aspect of controlling cooperative multi-agent direct networks, a hybrid system is a mix of discrete-time and continuous-time systems [12]. For cooperative relaying networks, the hybrid concept is employed for 
either the combination of different relay selection schemes or different relaying protocols. Using hybrid fullduplex (FD) and half-duplex (HD) relay selection scheme, authors in [13], [14] discovered the upper and lower bounds for OP of DF systems. Additionally, we can find a so-called hybridized power-time splittingbased relaying (HPTSR) protocol in [15]-[17] where the OP and maximum throughput were studied. Moreover, in [18], [19], the OP and outage capacity of a so-called joint time allocation and power splitting (JTAPS) scheme over the Rayleigh fading channels were studied concerning the statistical and instantaneous channel state information (CSI). Additionally, in [20], [21], the system performance in terms of the average channel capacity was investigated. We can find some other studies such as [22]-[25], that used the instantaneous CSI to determine the PS factor for a namely dynamic power splitting scheme (DPSS). The OP and throughput for multi-user cooperative relaying networks over Nakagami fading channels were studied in [26], [27]. Last but not least, [28]-[30] investigated the performance of time switching relaying (TSR) and power splitting relaying (PSR) protocols for dual-hop relaying networks in the indoor scenario modelled by log-normal fading channels.

It is noted that several existing studies were conducted mainly over outdoor scenarios which are modelled by Rayleigh and Nakagami-m fading methods. Indeed, there is scarcely recent studies regarding the SWIPT systems for indoor scenarios modelled by log-normal fading channels. Among them, there are papers [28], [29] where the HD AF and DF SWIPT networks were analyzed. Moreover, log-normal fading fits better for modelling shadowing effect from indoor moving objects and human bodies, as mentioned in [31]-[33]. Besides, as in [34], log-normal fading can be employed to model the small-scale fading of indoor ultrawideband (UWB) communications, and in [35]-[37] to model the short and long-term fading of slowlyvarying indoor channels. To overcome all of these constraints, it is essential to deploy cooperative relays for indoor networks, especially for internet of things (IoT) applications [38].

Thereby, in this paper, we study the performance of a hybrid TSR-PSR scheme over log-normal fading channels in a cooperative AF relaying network. We evaluate the system performance in terms of its outage behaviour. It is proven that the proposed hybrid protocol can deliver significantly lower $O P$ than the two separate protocols. Furthermore, we describe the system model in section 2 . In section 3 , we analyze the performance of the hybrid PSR-TSR AF system over log-normal fading channels. We report the simulation results and discuss them in section 4 . The conclusion is in section 5.

\section{SYSTEM MODEL}

Illustrated in Figure 1(a) (see in appendix) is the system model of a dual-hop network including a base station (BS), a relay (R) and a destination (D). Assumed without direct link, the BS-to-D communication can only be realized via R, with BS's transmission power of $P_{B}$. A part of the RF signal that BS sends is harvested by $\mathrm{R}$ for relaying task. We denote the BS-to-R, R-to-D distances, respectively, with $I_{1}$ and $I_{2}$, and according channel coefficients of $h_{1}$ and $h_{2}$. Additionally, we have two random variables (RVs) $\left|h_{1}\right|^{2}$ and $\left|h_{2}\right|^{2}$ which are independently and identically distributed (i.i.d.) over the block time following the log-normal distribution, respectively, with parameters $L N\left(\mu_{h 1}, \sigma_{h 1}^{2}\right)$ and $L N\left(\mu_{h 2}, \sigma_{h 2}^{2}\right)$. Moreover, we have the mean value of $10 \log \left(\left|h_{i}\right|^{2}\right)$ denoted as $\mu_{h i}$, and the standard deviation of $10 \log \left(\left|\sigma_{h i}\right|^{2}\right), i \in\{1,2\}$, denoted as $\sigma_{h i}^{2}$. BS-to-R and R-to-D have complex Gaussian background noise, $n_{R}$ and $n_{D}$, the former has zero mean and the latter has variance $N_{0}$. Last but not least, we have $m$ which is the path-loss exponent.

Considering a hybrid PSR-TSR in Figure 1(b) (see in appendix), we have a two-time-slot block time, $T$, which is split by TS factor, $\alpha_{2}$, to $\alpha_{2} T$ and $\left(1-\alpha_{2}\right) T$ given that $\alpha_{2} \in[0,1]$. In $\alpha_{2} T$, R receives signal from BS. We divide this 1 st time slot into two segments which are $\sqrt{\alpha_{1}}$ and $\sqrt{1-\alpha_{1}}$, with the PS factor, $\alpha_{1}$, given that $\alpha_{1} \in[0,1]$. We use the segment $\sqrt{\alpha_{1}}$ for RF EH, and the segment $\sqrt{1-\alpha_{1}}$ for receiving information from BS. Then, within the 2 nd time slot $\left(1-\alpha_{2}\right) T$, the harvested energy we have at $\mathrm{R}$ is used up to decode and forward the signal via the BS-to-D link. Besides, we assume that the processing power of R is neglected because it is relatively small in comparison with the transmission power of R-to-D [11], [39].

\section{PERFORMANCE ANALYSIS HYBRID PSR-TSR SCHEME}

Within the 1st time block, we have signal transmitted from BS to R for decoding given by (1).

$$
y_{r}=\sqrt{1-\alpha_{1}} \sqrt{P_{\mathrm{B}} l_{1}^{-m}} h_{1} s_{x}+n_{\mathrm{R}}
$$

where we normalize the transmitted signal from BS and denote it with $s_{x}, E=\left[\left|s_{x}\right|^{2}\right]=1$. Then, we have the received signal for $\mathrm{EH}$ in the 1st time slot as (2). 


$$
E=\eta \alpha_{2} T \alpha_{1} P_{B}\left|h_{1}\right|^{2} l_{1}^{-m},
$$

where there is the energy conversion efficiency $\eta, \eta \in(0,1)$.

Within the 2nd time slot, we have signal transmitted from R to D as (3).

$$
P_{R}=E /\left(1-\alpha_{2}\right) T=\alpha_{2} \eta \alpha_{1} P_{B}\left|h_{1}\right|^{2} l_{1}^{-m}\left(1-\alpha_{2}\right)^{-1}
$$

After the base-band processing and amplifying processes at $\mathrm{R}$, the $\mathrm{R}$ transmit signal is

$$
s_{r}=\sqrt{\frac{\left(1-\alpha_{1}\right) P_{B} P_{R}}{l_{1}^{m}}} G h_{1} s_{x}+\sqrt{P_{R}} G n_{R}
$$

where we have the R gain, $G$ of the HD-AF system as (5),

$$
G=\left(\sqrt{\frac{\left(1-\alpha_{1}\right) P_{B}}{l_{1}^{m}}} h_{1}^{2}+N_{0}\right)^{-1} \approx\left(\sqrt{\frac{\left(1-\alpha_{1}\right) P_{B}}{l_{1}^{m}}} h_{1}^{2}\right)^{-1}
$$

From (5), we can formulate the received signal at D as (6).

$$
y_{d}=\sqrt{\frac{P_{\mathrm{R}}}{l_{2}^{m}}} h_{2} s_{r}+n_{\mathrm{D}}=\sqrt{\frac{\left(1-\alpha_{1}\right) P_{B} P_{R}}{l_{1}^{m} l_{2}^{m}}} G h_{1} h_{2} s_{x}+\sqrt{\frac{P_{R}}{l_{2}^{m}}} G h_{2} n_{R}+n_{D} .
$$

Consequently, we substitute (3) and (5) into (6) then manipulate it to get the signal-to noise ratio $(\mathrm{SNR})$ at $\mathrm{D}$ as (7),

$$
\gamma_{D}=\frac{a_{1}\left|h_{1}\right|^{2}\left|h_{2}\right|^{2}}{a_{2}\left|h_{2}\right|^{2}+a_{3}}
$$

where $a_{1}=\eta \alpha_{1} \alpha_{2}\left(1-\alpha_{1}\right) \chi, a_{2}=\eta \alpha_{1} \alpha_{2} l_{1}^{m}, a_{3}=\left(1-\alpha_{1}\right)\left(1-\alpha_{2}\right) l_{1}^{m} l_{2}^{m}$, and $\chi=P_{S} / N_{0}$.

We evaluate the system performance by analyzing the achievable date rate and the $O P$. Therefore, firstly, we derive the achievable data rate at $\mathrm{D}$ as (8).

$$
R_{D}=\frac{1}{2}\left(1-\alpha_{2}\right) \log _{2}\left(1+\gamma_{D}\right)
$$

where we have the HD relaying factor of $1 / 2$.

The probability that the instantaneous data transmission rate declines below the source transmission rate of $R_{0}=(1 / 2)\left(1-\alpha_{2}\right) \log _{2}\left(1+\gamma_{0}\right)$ ( bits/sec/Hz), with a pre-specified threshold value of $\gamma_{0}$, is named OP. We can obtain the OP at D for the AF hybrid PSR-TSR systems as (9),

$$
\mathcal{O P}=\operatorname{Pr}\left(\gamma_{D}<\gamma_{0}\right)
$$

where $\gamma_{0}=2^{2 R_{0} /\left(1-\alpha_{2}\right)}-1$, and $\operatorname{Pr}($.$) is the probability function. We can express the OP analytically as (10).$

$$
O P=1-\frac{10}{\operatorname{In}(10) \sqrt{8 \pi \sigma_{h_{1}}^{2}}} \int_{\gamma_{0} \alpha_{2}}^{\infty} \frac{1}{x} \varrho\left(\frac{\frac{10}{\operatorname{In}(10)} \operatorname{In}(\vartheta(x))-2 \mu_{h_{2}}}{2 \sigma_{h_{2}}}\right) \times \exp \left(\frac{\left(\frac{10}{\operatorname{In}(10)} \operatorname{In}(\vartheta(x))-2 \mu_{h_{2}}-\frac{10}{\ln (10)} \ln \left(\alpha_{1}\right)\right)^{2}}{8 \mu_{h_{1}}^{2}}\right)
$$

where $\vartheta(x)=\gamma_{0} a_{3} /\left(x-\gamma_{0} a_{2}\right)$.

Proof:

With (7) and (9), we can formulate the OP for the HD-AF hybrid PSR-TSR network as (11). 


$$
\mathcal{O P}=\operatorname{Pr}\left(\left|h_{2}\right|^{2}<\frac{a_{3} \gamma_{0}}{a_{1}\left|h_{1}\right|^{2}-a_{2} \gamma_{0}}\right)
$$

Since $\left|h_{2}\right|^{2}$ is always a positive value, we can re-express the probability as (12).

$$
O P= \begin{cases}\operatorname{Pr}\left(\left|h_{2}\right|^{2}>\frac{\gamma_{0} \alpha_{2}}{\alpha_{1}\left|h_{1}\right|^{2}-\gamma_{0} \alpha_{3}}\right)=1, & \left|h_{1}\right|^{2} \geq \frac{\gamma_{0} \alpha_{3}}{\alpha_{1}} \\ \operatorname{Pr}\left(\left|h_{2}\right|^{2}<\frac{\gamma_{0} \alpha_{2}}{\alpha_{1}\left|h_{1}\right|^{2}-\gamma_{0} \alpha_{3}}\right), \quad\left|h_{1}\right|^{2}<\frac{\gamma_{0} \alpha_{3}}{\alpha_{1}}\end{cases}
$$

Thus, we rewrite the OP in (12) as (13).

$$
\mathcal{O P}=\int_{0}^{\gamma_{0} a_{3} / a_{1}} f_{\left|h_{1}\right|^{2}}(x) \mathrm{d} x+\int_{\gamma_{0} a_{3} / a_{1}}^{\infty} f_{\left|h_{1}\right|^{2}}(x) \operatorname{Pr}\left(\left|h_{2}\right|^{2} \leq \frac{\gamma_{0} a_{3}}{a_{1} x-\gamma_{0} a_{2}}\right) \mathrm{d} x
$$

We express the probability density function (PDF), $f_{\left|h_{1}\right|^{2}}(x)$, and the cumulative distribution function (CDF), $F_{\left|h_{2}\right|^{2}}(x)$, of the two RVs $\left|h_{1}\right|^{2},\left|h_{2}\right|^{2}$, respectively, as (14),

$$
f_{\left|h_{1}\right|^{2}}(x)=\frac{10}{\ln (10) x \sqrt{8 \pi \sigma_{h_{1}}^{2}}} \exp \left(-\frac{\left(\frac{10}{\ln (10)} \ln (x)-2 \mu_{h_{1}}-\frac{10}{\ln (10)} \ln \left(a_{1}\right)\right)^{2}}{8 \sigma_{h_{1}}^{2}}\right),
$$

and

$$
F_{\left|h_{2}\right|^{2}}(\vartheta(x))=1-\varrho\left(\frac{\frac{10}{\operatorname{In}(10)} i n(\vartheta(x))-2 \mu_{h 2}}{2 \sigma_{h 2}}\right)
$$

where following the same in [26], [28], with the Gaussian Q-function, $\varrho(x)=\int_{x}^{\infty} \frac{1}{\sqrt{2 \pi}} \exp \left(-\frac{t^{2}}{2}\right) d t$

Substituting (14) and (15) into (13), we obtain the OP of the HD hybrid PSR-TSR network in (10). This is the end of the proof.

\section{RESULTS AND DISCUSSION}

In this section, we investigate the impact of the TS factor, the PS factor and SNR on the OP of the hybrid PSR-TSR systems over the log-normal fading channel via the Monte Carlo simulation results of the previously derived expressions. We use the following system parameters in the simulations as shown in Table 1 unless specified otherwise.

Table 1. Parameter setting

\begin{tabular}{cccc}
\hline Symbol & Value & Symbol & Value \\
\hline$P_{B}$ & 1 (Walts) & $\sigma_{h 1}=\sigma_{h 2}$ & $4(\mathrm{~dB})$ \\
$\eta$ & 1 & $\mu_{h 1}=\mu_{h 2}$ & $3(\mathrm{~dB})$ \\
$M$ & 2 & $l_{1}=l_{2}$ & $5(\mathrm{~m})$ \\
$N_{0}$ & 1 & $R_{0}$ & $2(\mathrm{bps} / \mathrm{Hz})$ \\
\hline
\end{tabular}

Figures 2 and 3 depict the OP of the hybrid PSR-TSR systems versus, respectively, TS factor of $\alpha_{2}$, and PS factor of $\alpha_{1}$. As in Figure 2, if we fix $\alpha_{1}=0.5$ and vary $\alpha_{2}$, we will obtain the lowest curve which yields the best system performance. It is interesting that all the curves in Figure 2 reach their optimal values at $\alpha_{2}=0.25$. On the other hand, as illustrated in Figure 3, for fixed $\alpha_{2}=0.5$ and varied $\alpha_{1}$, the curve is the highest indicating the worst system performance. The optimal values for the curves in Figure 3 distributed 
from $\alpha_{1}=0.7$ to 0.9 . Another obvious trait for both figures is that as TS and PS factors approach either zero or one, we have a $100 \%$ probability of system shutdown. This is because as $\alpha_{2}$ and $\alpha_{1}$ are too small or too large during the 1st time slot, the transmit power from EH process in the 2nd time slot becomes, respectively, insufficient or unnecessarily large leading to poor system capacity [28].

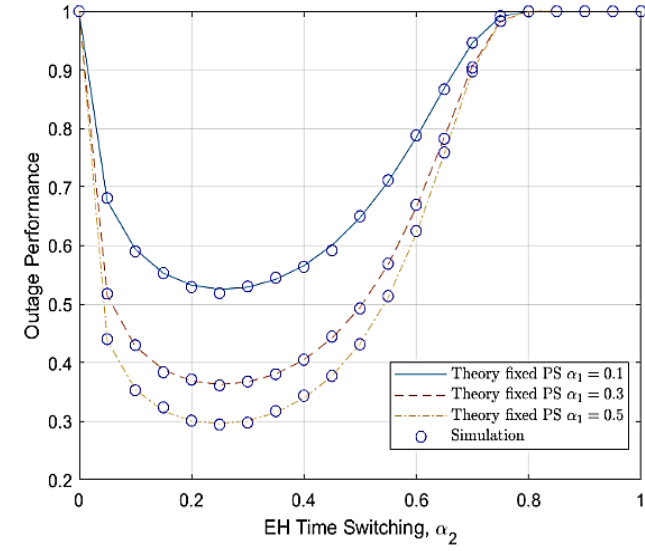

Figure 2. Outage performance versus the EH TS factor, $\alpha_{2}$

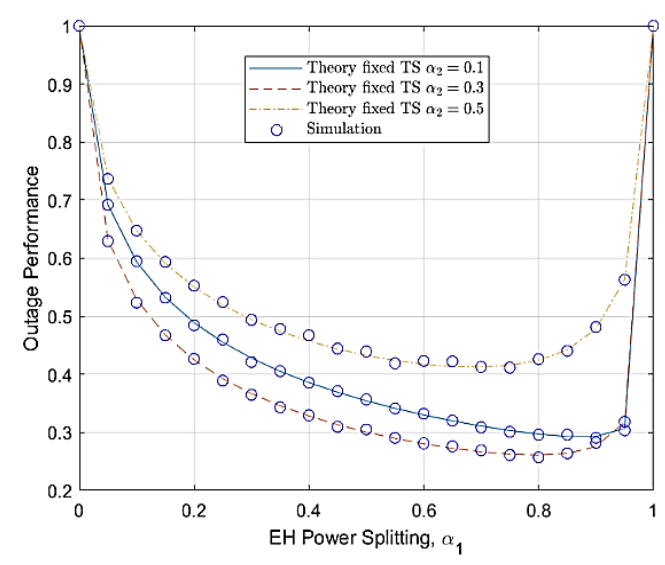

Figure 3. Outage performance versus the EH PS factor, $\alpha_{1}$

Besides, Figures 4 and 5 plot the OP versus the SNR, $\chi$, with, respectively, three pairs of EH PS and TS factors, i.e., $\left(\alpha_{2}=0.3, \alpha_{1}=0.5\right),\left(\alpha_{2}=0.3, \alpha_{1}=0.3\right),\left(\alpha_{2}=0.5, \alpha_{1}=0.3\right)$, and two data transmission rates, i.e., $R_{0}=2$ (bps/Hz), and $R_{0}=4(\mathrm{bps} / \mathrm{Hz})$. In general, we can see that the OP decreases as the SNR increases. The hybrid PSR-TSR scheme with higher TS factor performs better. Additionally, the OP gets worse as the transmission rate grows because the power for $\mathrm{EH}$ and the transmission time decrease in the 2nd slot [11]. Generally speaking, the simulation results and theory agree well with each other. Thereby, we can use the derived expressions for further studies.

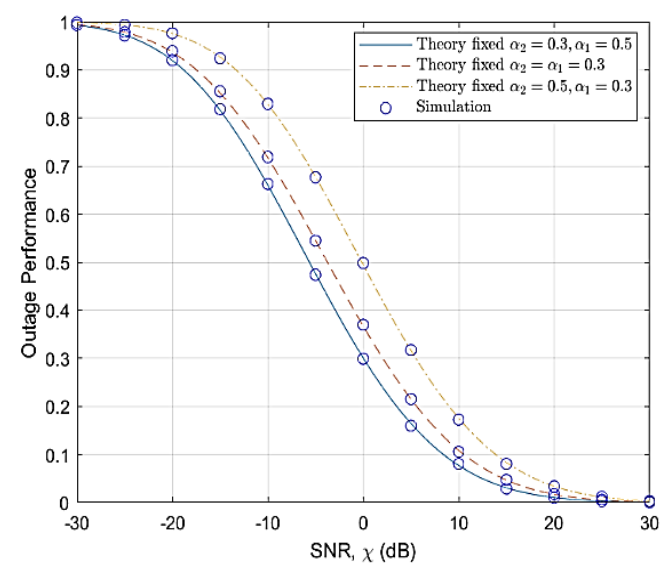

Figure 4. OP versus SNR under various dynamic switching condition

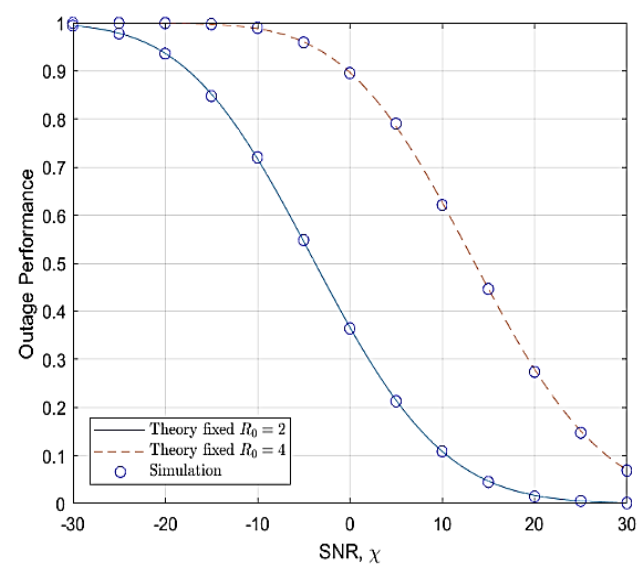

Figure 5. OP versus SNR with various data transmission rate

\section{CONCLUSION}

To summarize, we study a namely hybrid PSR-TSR protocol employed in a cooperative EH AF relaying network over log-normal fading channels by assessing the analytical expression of the OP. From the simulation results, we are able to conclude that the hybrid PSR-TSR scheme remarkably outperforms the standalone PSR and TSR schemes. Moreover, we prove that the system performance can be raised in association with the increase of EH TS factor and the decrease in data transmission rate. 


\section{APPENDIX}

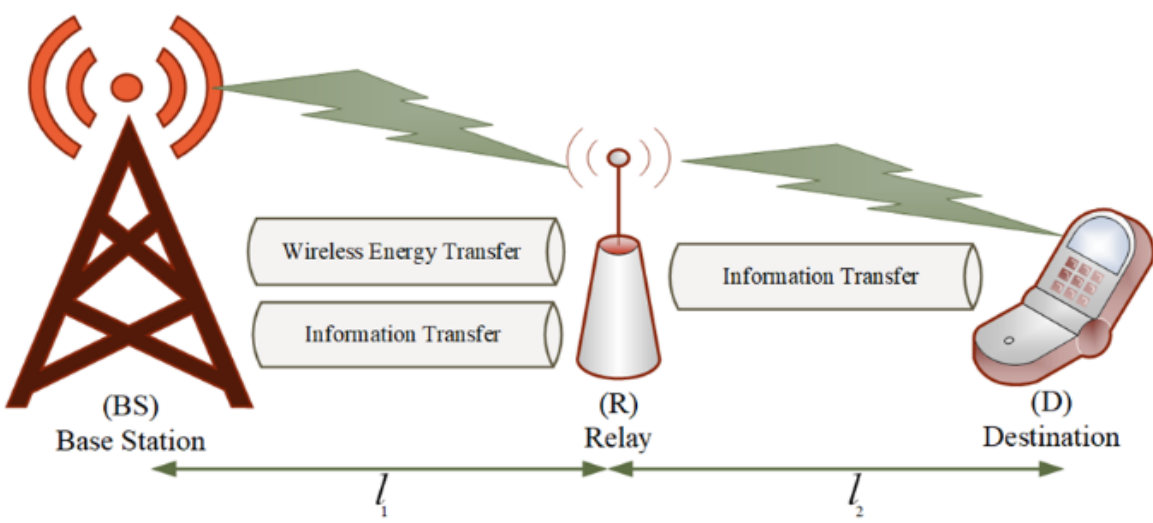

(a)

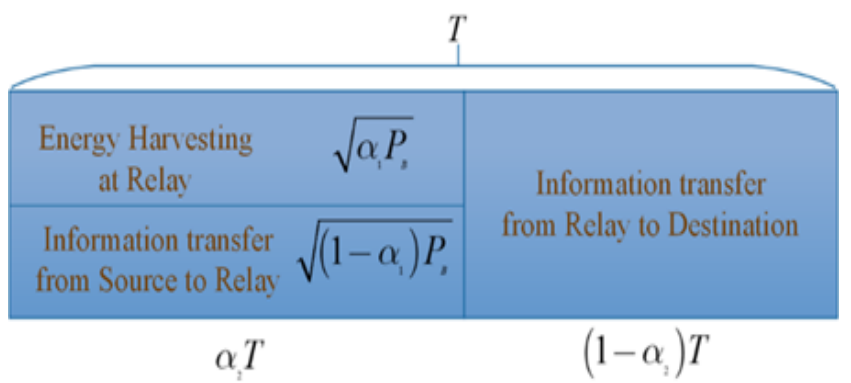

(b)

Figure 1. This figure are; (a) system model, (b) hybrid PSR-TSR protocol

\section{ACKNOWLEDGEMENTS}

Thanks for the Saigon International University (SIU) funds supporting this project. This research is funded by Thu Dau Mot University under grant number DT.20.2-020.

\section{REFERENCES}

[1] A. A. Nasir, X. Zhou, S. Durrani and R. A. Kennedy, "Relaying Protocols for Wireless Energy Harvesting and Information Processing," IEEE Transactions on Wireless Communications, vol. 12, no. 7, pp. 3622-3636, 2013, doi: 10.1109/TWC.2013.062413.122042.

[2] M. H. Misran, S. K. A. Rahim, M. A. M. Said, M. A. Othman, "A systematic optimization procedure of antenna miniaturization for efficient wirelessenergy transfer," International Journal of Electrical and Computer Engineering (IJECE), vol. 9, no 4, pp. 3159-3166, 2019, doi: 10.11591/ijece.v9i4.pp3159-3166

[3] K. Rabie, B. Adebisi and M. Alouini, "Half-Duplex and Full-Duplex AF and DF Relaying With Energy-Harvesting in Log-Normal Fading," IEEE Transactions on Green Communications and Networking, vol. 1, no. 4, pp. 468-480, 2017, doi: 10.1109/TGCN.2017.2740258.

[4] T. N. Nguyen, P. Tran, H.-Sy Nguyen, D.-T. Do, "On the Performance of a Wireless Powered Communication System Using a Helping Relay,” Radioengineering, vol. 26, no. 3, pp. 860-869, 2017, doi: 10.13164/re.2017.0860.

[5] H. -S. Nguyen, T.-S. Nguyen, \& M. Voznak, "Wireless powered D2D communications underlying cellular networks: design and perfor-mance of the extended coverage," Automatika, vol 58, no. 4, pp. 391-399, 2018, doi: 10.1080/00051144.2018.1455016.

[6] H. -S. Nguyen, T. T. Hoc Ly, T.-S. Nguyen, V. V. Huynh, T.-L. Nguyen, and M. Voznak, "Outage performance analysis and SWIPT optimization in energy-harvesting wirelesssensor network deploying NOMA," Sensors, vol. 19, no. 3, 2019, Art. No. 613, doi: 10.3390/s19030613

[7] H. -S. Nguyen, M. Vozňák, M.-Tung Nguyen, L. Sevcik, "Performance Analysis with Wireless Power Transfer Constraint Policies in Full-Duplex Relaying Networks," Elektron Elektrotech, vol. 23, no. 4, pp. 70-76, 2017, doi: 10.5755/j01.eie.23.4.18725. 
[8] O. Kazuma, K. Yamaguchi, K. Iida, "Wireless power transfer using multiple-transmitters for high stability for position," International Journal of Electrical and Computer Engineering (IJECE), vol. 10, no 3, pp. 2245-2249, 2020, doi: 10.11591/ijece.v10i3.pp2245-2249.

[9] R. Tao, A. Salem and K. A. Hamdi, "Adaptive Relaying Protocol for Wireless Power Transfer and Information Processing," IEEE Communications Letters, vol. 20, no. 10, pp. 2027-2030, 2016.

[10] H. -S. Nguyen, D.-T. Do, A.-H. Bui, M. Vozňák, "Self-Powered Wireless Two-Way Relaying Networks: Model and Throughput Performance with Three Practical Schemes," Wireless Personal Communications, vol. 97, no. 11, pp. 613-631, 2017.

[11] H. -S. Nguyen, Dinh-T. Do, M. Voznak, "Two-way relaying networks in green communications for 5G: Optimal throughput andtrade off between relay distance on power splitting-based and time switching-based relaying SWIPT," AEU-International Journal of Electronics and Communications, vol. 70, no. 12, pp. 1637-1644, 2016, doi: 10.1016/j.aeue.2016.10.002.

[12] Y. Shang, "Consensus of Hybrid Multi-Agent Systems with Malicious Nodes," IEEE Transactions on Circuits and Systems II: Express Briefs, vol. 67, no. 4, pp. 685-689, 2020, doi: 10.1109/TCSII.2019.2918752.

[13] F. Wang, W. Xu, S. Li, Z. Feng and J. Lin, "Outage Probability Analysis of DF Relay Networks with RF Energy Harvesting," 2015 IEEE Global Communications Conference (GLOBECOM), San Diego, CA, USA, 2015, pp. 1-5, doi: 10.1109/GLOCOM.2015.7417579.

[14] H. -S. Nguyen, T.-S. Nguyen, V.-Tri Vo, M. Voznak, "Hybrid full-duplex/half-duplex relay selection scheme with optimal power under individual power constraints and energy harvesting," Computer Communications, vol. 124, pp. 31-44, 2018, doi: 10.1016/j.comcom.2018.04.014

[15] F. Ojo, and M. F. Mohd Salleh, "Throughput Analysis of a Hybridized Power Time Splitting Based Relaying Protocol for Wireless Information and Power Transfer in Cooperative Networks," IEEE Access, vol. 6, pp. 24137-24147, 2018, doi: 10.1109/ACCESS.2018.2828121.

[16] H. -S. Nguyen, A. Bui, D. Do and M. Voznak, "Imperfect channel state information of AF and DF energy harvesting cooperative networks," China Communications, vol. 13, no. 10, pp. 11-19, 2016, doi: 10.1109/CC.2016.7732008.

[17] H. -S. Nguyen, D.-T. Do, T.-S. Nguyen, \& M. Voznak, "Exploiting hybrid time switching-based and power splitting-based relaying protocol inwireless powered communication networks with outdated channel state information," Automatika, vol 58, no. 1, pp. 111-118, 2017, doi: 10.1080/00051144.2017.1372124

[18] D. Wang, Y. Li, Y. Ye, H. Xia and H. Zhang, "Joint Time Allocation and Power Splitting Schemes for DF Energy Harvesting Relaying Networks," 2017 IEEE 86th Vehicular Technology Conference (VTC-Fall), Toronto, ON, Canada, 2017, pp. 1-5, doi: 10.1109/VTCFall.2017.8288115.

[19] Y. Ye, Y. Li, D. Wang, F. Zhou, R. Q. Hu and H. Zhang, "Optimal Transmission Schemes for DF Relaying Networks Using SWIPT," IEEE Transactions on Vehicular Technology, vol. 67, no. 8, pp. 7062-7072, 2018, doi: 10.1109/TVT.2018.2826598.

[20] H. S. Nguyen, N. X. H. Nguyen, Q. P. Ma, J. Jalowiczor, M. Voznak. "Symbol Error Probability of Secondary User in Underlay Cognitive Radio Networks with Adaptive Transmit Power Constraint". In Proc. of Multimedia Communications, Services and Security. (MCSS 2020), vol. 1284, 2020. doi: 10.1007/978-3-030-59000-0_23

[21] N. Kumar and V. Bhatia, "Outage Probability and Average Channel Capacity of Amplify-and-Forward in Conventional Cooperative Communication Networks over Rayleigh Fading Channels," Wireless Personal Communications, vol. 88, no. 4, pp. 943-951, 2016, doi: 10.1007/s11277-016-3221-0.

[22] Y. Ye, Y. Li, F. Zhou, N. Al-Dhahir and H. Zhang, "Power Splitting-Based SWIPT with Dual-Hop DF Relaying in the Presence of a Direct Link," IEEE Systems Journal, vol. 13, no. 2, pp. 1316-1319, 2019, doi: 10.1109/JSYST.2018.2850944.

[23] N. T. Do, D. B. da Costa, T. Q. Duong, V. N. Q. Bao and B. An, "Exploiting Direct Links in Multiuser Multirelay SWIPT Cooperative Networks With Opportunistic Scheduling," IEEE Transactions on Wireless Communications, vol. 16, no. 8, pp. 5410-5427, 2017, doi: 10.1109/TWC.2017.2710307.

[24] Y. Yinghui, Y. Li, L. Shi, R. Q. Hu and H. Zhang, "Improved Hybrid Relaying Protocol for DF Relaying in the Presence of a Direct Link," IEEE Wireless Communications Letters, vol. 8, no. 1, pp. 173-176, 2019, doi: 10.1109/LWC.2018.2865476.

[25] Y. Ye, L. Shi, X. Chu, H. Zhang and G. Lu, "On the Outage Performance of SWIPT-Based Three-Step Two-Way DF Relay Networks," IEEE Transactions on Vehicular Technology, vol. 68, no. 3, pp. 3016-3021, 2019, doi: 10.1109/TVT.2019.2893346.

[26] Y. Feng, V. C. M. Leung and F. Ji, "Performance Study for SWIPT Cooperative Communication Systems in Shadowed Nakagami Fading Channels," IEEE Transactions on Wireless Communications, vol. 17, no. 2, pp. 1199-1211, 2018, doi: 10.1109/TWC.2017.2776933.

[27] Y. Meng, V. C. M. Leung and F. Ji, "Transmission Performance Analysis of Cognitive NOMA with SWIPT over Nakagami-m FadingChannels," IEEE 19th International Conference on Communication Technology (ICCT), Xi'an, China, pp. 770-775, 2019.

[28] K. Rabie, A. Salem, E. Alsusa and M. Alouini, "Energy-harvesting in cooperative AF relaying networks over lognormal fading channels," 2016 IEEE International Conference on Communications (ICC), Kuala Lumpur, Malaysia, 2016, pp. 1-7, doi: 10.1109/ICC.2016.7511559

[29] K. Rabie, B. Adebisi and M. Alouini, "Wireless Power Transfer in Cooperative DF Relaying Networks with LogNormal Fading," 2016 IEEE Global Communications Conference (GLOBECOM), Washington, DC, USA, 2016, pp. 1-6, doi: 10.1109/GLOCOM.2016.7842388. 
[30] V. -V. Huynh, H.-SyNguyen, Ly T. T. Hoc, T.-S. Nguyen, M. Voznak, "Optimization issues for data rate in energy harvesting relay-enabled cognitive sensor networks," Computer Networks, vol. 157, pp. 29-40, 2019, doi: 10.1016/j.comnet.2019.04.012.

[31] H. Hashemi, "The indoor radio propagation channel," Proceedings of the IEEE, vol. 81, no. 7, 1993, pp. 943-968, doi: $10.1109 / 5.231342$.

[32] S. Y. Seidel, K. Takamizawa and T. S. Rappaport, "Application of second-order statistics for an indoor radio channel model," IEEE 39th Vehicular Technology Conference, San Francisco, CA, USA, vol. 2, 1989, pp. 888-892, doi: 10.1109/VETEC.1989.40179.

[33] M. -S. Alouini, and M. K. Simon, "Dual diversity over correlated log-normal fading channels," IEEE Transactions on Communications, vol. 50, no. 12, pp. 1946-1959, 2002 doi: 10.1109/TCOMM.2002.806552.

[34] F. Heliot, X. Chu, R. Hoshyar and R. Tafazolli, "A tight closed-form approximation of the log-normal fading channel capacity," IEEE Transactions on Wireless Communications, vol. 8, no. 6, pp. 2842-2847, 2009, doi: 10.1109/TWC.2009.080972.

[35] M. Safari and M. Uysal, "Cooperative diversity over log-normal fading channels: performance analysis and optimization," IEEE Transactions on Wireless Communications, vol. 7, no. 5, pp. 1963-1972, 2008, doi: 10.1109/TWC.2008.070393.

[36] A. Kalantari, M. Mohammadi and M. Ardebilipour, "Performance analysis of opportunistic relaying over imperfect non-identical log-normal fading channels," 2011 IEEE 22nd International Symposium on Personal, Indoor and Mobile Radio Communications, Toronto, ON, Canada, 2011, pp. 1909-1913, doi: 10.1109/PIMRC.2011.6139842.

[37] P. Varzakas, "Average Channel Capacity for Rayleigh Fading Spread Spectrum MIMO systems," International Journal of Communication Systems, vol. 19, no. 10, pp. 1081-1087, 2006, doi: 10.1002/dac.784.

[38] Z. Chen, L. Wang, D. Han and H. Zeng, "A Unified Performance Analysis of Relaying Communication System for IoT Application with Hybrid Fading," IEEE Internet of Things Journal, vol. 7, no. 1, pp. 570-583, 2020, doi: 10.1109/JIOT.2019.2951179 .

[39] Y. Liu, R. Xiao, J. Shen, H. Yang, "Hybrid protocol for wireless energy harvesting network over log-normal fading channel,” The Journal of Engineering, vol. 2018, no. 6, pp. 339-341, 2018, doi: 10.1049/joe.2017.0892. 